\title{
El perfil laboral y educativo de los jóvenes psicólogos
}

\section{Job and educational profile of young psychologists}

\author{
Denise Benatuil ${ }^{1}$ y Juliana Laurito \\ Universidad de Palermo, Argentina
}

(Recepción: Octubre 2009 - Aceptación: Diciembre 2009)

\begin{abstract}
Resumen
En este trabajo se analiza la inserción ocupacional de una muestra de jóvenes profesionales egresados de la Licenciatura en Psicología. Se trata de una temática de gran relevancia y poco abordada hasta la actualidad. Su estudio permite conocer la situación de los jóvenes profesionales hoy y su grado de inserción laboral. Este estudio contribuye a la comprensión de las características propias de un campo profesional singular, con rasgos distintivos. A su vez los datos arrojados tienen un alto grado de trasferencia ya que aportan información para evaluar y repensar la formación actual del Psicólogo en la Argentina, considerando sus fortalezas y debilidades, analizando los logros y las deudas pendientes.
\end{abstract}

Palabras clave: Jóvenes psicólogos - Inserción laboral- Formación de postgrado.

\begin{abstract}
This paper analyzes the occupational structure of a sample of young professionals graduated in Psychology. This is a relevant topic and with a few studies until now. This studied allow knowing the situation of young professionals today, the level of employability. This study contributes to understanding the characteristics of a particular field, with distinctive features. In turn, the data, have a high degree of transference because they provide information to evaluate and rethink the current training Psychologist in Argentina, considering their strengths and weaknesses, analyzing the achievements and outstanding debts.

Key words: Young psychologists - Job placement - Postgraduate education.
\end{abstract}

1 Correspondencia: Dra. Denise Benatuil. Dirección: Av. Cabildo 66 5² Ciudad de Buenos Aires CP 1426 Email: dbenatuil@ iname.com Teléfono: (+54911) 57958282 Institución: Universidad de Palermo 


\section{La relación entre la educación y el trabajo}

La relación entre formación universitaria y mercado productivo ha comenzado a generar gran interés en los últimos años. El informe del Banco Mundial "Higher Education: Lessons of Experien$c e$ " (1994) señala que un elemento clave en la crisis actual del sistema se basa en las tensiones entre la educación superior y el empleo. También la OIT destaca que frente al proceso de globalización, las áreas educativas y el entrenamiento para el trabajo, son dos de los grandes desafíos. Desde comienzos de los 90` la OECD se encuentra realizando diferentes proyectos de investigación sobre la transición entre la educación y el ingreso al mercado laboral (OECD, 1992,1994).

Lo expuesto anteriormente, denota que la relación entre la formación universitaria y el mercado laboral ha generado un gran interés y producción científica a nivel mundial. Son varias las investigaciones que abordan dicha temática. A continuación se sintetizan algunas cuestiones que resultan relevantes:

I) La educación universitaria y el mundo del trabajo tienen tiempos y objetivos diferentes. El trabajo se modifica en base a la innovación tecnológica, cambios abruptos, surgimiento de nuevas necesidades y demandas. Desde la educación son varias las estrategias posibles de articulación con el sistema laboral, pero la trasmisión de conocimientos demanda procesos prolongados de tiempo y sus modificaciones son lentas y graduales, por lo que tiende a generarse un desequilibrio entre ambas esferas (Casali, Farber, Giangiácomo, Hernández \& Toribio, 2005).

II) Es necesario identificar las diversas demandas sociales y del mercado laboral específico del país o región para poder elaborar políticas, programas y acciones destinadas a la formación para el trabajo (Riquelme \& Herger, 2002).

III) En la actualidad, se destaca el rol de la educación en los procesos de incorporación al mundo global. En especial se ha enfatizado el papel determinante de la educación como factor clave para el desarrollo económico y a partir de allí se han desencadenado procesos de reforma de los sistemas educativos (Testa \& Spinoza, 2005).

IV) Se han planteado una serie de interrogantes que surgen de esta estrecha pero a su vez tensa relación y que resultan orientadoras para reflexionar: ¿Qué perfiles de graduados requiere el mundo laboral? ¿La universidad ha realizado modificaciones en los programas y contenidos curriculares ajustados a los cambios de los paradigmas productivos? ¿La universidad, cumple un papel de productora de recursos humanos para el mercado laboral? ¿La universidad tendría que tener una postura crítica y de construcción de conocimientos sin tener en cuenta el ajuste con las demandas del mercado? ¿Cuáles son las nuevas tendencias del mercado de trabajo en relación a los empleo de los egresados universitarios? ¿Obtener un título de grado es garantía de movilidad social? (Pancenza, 1997).

\section{La transición de estudiante a profesional}

La formación del profesional comienza en la Universidad, lugar donde se adquieren los conocimientos básicos, que permiten desarrollarse en el área de competencia. Aunque al terminar los estudios y obtener el título de grado, están habilitados para desempeñar su profesión, se reconoce un momento entre el fin de los estudios y la inserción profesional, en que el graduado es demasiado inexperto para ejercer su rol, pero ya no pertenece al mundo estudiantil. Se encuentra en un período de transición hasta consolidarse como profesional (Richino, 1985).

La inserción laboral esta determinada por las competencias que se adquieren en la formación de grado, es decir, el nivel de ajuste entre los saberes enseñados en la Universidad y lo requerido en el mercado laboral va a ser sumamente influenciable en la inserción del joven profesional. El comienzo de la vida laboral y el traspaso de la etapa estudiantil a la de profesional es a través de pequeños acercamientos, comenzando por puestos laborales poco estables (Pedroza Flores, Villalobos Monroy \& Morales Euzarraga, 2007). 
Se sostiene que en la acción se forman y desarrollan los roles, donde el graduado emprende la tarea de aprender a ser (Richino, 1985). Se conoce como proceso de socialización profesional al aprendizaje de roles, valores, responsabilidades y actitudes que son relevantes para la profesión. No hay un libro de texto o manual, ni puede trasferirse la experiencia mediante la enseñanza verbal en una clase áulica. Se aprende mediante la experiencia, en el ejercicio profesional, en reuniones de equipo y debates con otros profesionales (Ortega Álvarez, 1982).

Se pueden señalar algunos aspectos que interfieren en la relación entre formación de grado y empleo profesional: I) para el trabajo se requieren nuevas competencias que no siempre están contempladas en la formación, II) la diversidad de empleos excede los campos de inserción clásicos, que suelen ser los desarrollados a lo largo de la formación, III) es frecuente hallar un desajuste entre ocupación y cualificación, IV) en la actualidad, se ha producido una devaluación de los títulos de grado, siendo cada vez más necesaria la formación de postgrado, V) la recesión económica suele generar nuevas demandas y mayores niveles de precariedad laboral (Pedroza Flores, et al., 2007).

Los egresados, al salir al mercado laboral, ponen a prueba su formación, evaluando si esta se adecua y si está a la altura o no de las demandas del mercado (Pedroza Flores, et al. 2007). Por lo que cada vez se le da más relevancia a la necesidad de realizar prácticas preprofesionales ya que en el caso de la carrera de psicología, en Argentina la matrícula profesional es habilitante y para solicitarla solo se requiere presentar el título de grado. Por este motivo es necesario que el profesional tenga algún contacto con el ejercicio profesional de forma supervisada y no un salto cuali y cuantitativo de la formación exclusivamente teórica a la completa habilitación para el libre ejercicio profesional en todos los campos del quehacer profesional.

\section{La inserción en países de América Latina}

En América Latina la Psicología se ha constituido como profesión en los últimos 50 años, en la mayoría de los países existen facultades de Psicología con una gran cantidad de alumnos y egresados (Ardila, 2004). En Brasil se estiman 156.000 psicólogos, en la Argentina 55.853, en Colombia 15.000, en México 100.000 y en Chile 7.500 (Alonso, 2005).

En relación al área de inserción profesional, tiene algunas variaciones según el país, sin embargo los campos tradicionales como Psicología Clínica, Educacional, Social y Laboral siguen teniendo prioridad. También se observa un crecimiento de áreas como la Psicología Jurídica, de la Salud, del Deporte, Ecológica y Ambiental, mostrando variación en el desarrollo del área según la nación (Ardila, 2004).

A continuación se presentaran una serie de investigaciones de diferentes países de América Latina en relación a la temática, para poder dilucidar similitudes y diferencias con los resultados hallados en la Argentina. Se han seleccionado los países que tienen mayor cantidad de investigaciones sobre la inserción profesional de los jóvenes psicólogos.

Diferentes investigaciones con respeto a este tema en Chile sintetizan que el perfil se basa en un predominio de mujeres, satisfechos con su trabajo, cuyo ámbito laboral de preferencia es el área clínica, social y comunitaria. La mitad de la muestra se desempeña profesionalmente en dos lugares, y muy pocos tienen estudios de post grado (Miranda Zapata \& Sutulov Baez, 2007; Linn, 2007; Rojas 2007).

En México el perfil profesional se compone de un 85\% mujeres con una media de edad 31.53 años, el 90\% trabaja como psicólogo el 50\% está muy satisfecho con su trabajo (Huerta González, Valenzuela \& Rodríguez Llanes, 2007) El promedio de ocupación laboral de los egresados es alrededor del 75\%, de ellos el 50\% realizan actividades propias de su formación profesional, el 40\% en actividades medianamente relacionadas y el resto se desempeña en otras actividades (VerdugoLucero, Monroy-Galindo, Márquez-González \& Ceja-Castillo, 2002).

La situación en el país limítrofe de Brasil es similar, la psicología clínica más allá del paso de los años sigue ocupando el lugar central de inserción profesional de los psicólogos. En la actividad profesional de los psicólogos prevalece el desempeño laboral dentro del ámbito de lo estrictamente 
individual. Como ejemplo solo el 1.7\% desarrolla su labor profesional en actividades relacionadas con la psicología social (Moura, 2003).

En relación a la inserción de la psicología en la sociedad colombiana, Ardila (2004) sostiene que todavía se identifica al psicólogo con el profesional que se desempeña en el área clínica en el trabajo de consultorio. No existe un gran conocimiento por parte de la sociedad de otras áreas de inserción laboral, como son la investigación, el ámbito comunitario, como peritos en los ámbitos jurídicos y forenses, en instituciones y empresas y en los distintos niveles educativos. Por lo que considera que los psicólogos deben mostrar una imagen profesional clara y amplia de su campo de aplicación disciplinar para de esta forma poder ampliar su inserción.

\section{La inserción laboral del Psicólogo en la Argentina}

La inserción laboral se considera un hecho relevante para el individuo, ya que supone el encuentro con el trabajo y el desafío de un nuevo rol social. Constituye una de las transiciones más importantes en la vida de una persona (Peiró, 1991).

En la Argentina existe un incremento de jóvenes profesionales con dificultades para insertarse laboralmente (Castro Solano, 2004). Los cambios en los procesos económicos y productivos de los últimos años han revolucionado dos esferas institucionales centrales, como son el trabajo y la educación (Gómez, 2000).

La educación universitaria en épocas pasadas garantizaba la pronta inserción en el mercado laboral, situación que no ocurre hoy en día. Los egresados se encuentran sujetos a los cambios económicos que impactaron fuertemente en la posibilidad de ingresar en el mercado de trabajo; la creciente desocupación, la cantidad de profesionales altamente capacitados, la subocupación, la incertidumbre sobre la continuidad laboral, los contratos precarios, inestables, ilegales y las personas que se incorporan al mercado en puestos que requieren una formación o experiencia menores de las que tienen produciéndose un desajuste entre el nivel educativo y las herramientas requeridas para el puesto de trabajo (Isorini, Cerro \& Goni, 2006; Pancenza, 1997; Weisgras, 2006).

En relación a la tasa de desocupación de los jóvenes graduados se puede observar que en 1990 era del 2\%, en 1997 del 6\% y en el año 2000 alcanzo un 9\%. Este aumento se vio acelerado debido a los crecientes problemas de la estructura económica para absorber el incremento en la oferta de empleo de personas con altos niveles educativos (Gómez, 2000).

En relación al campo profesional del psicólogo, existe gran número de psicólogos en la Argentina. Según datos que revela una investigación realizada por Alonso hay 154 psicólogos cada 100.000 habitantes, si se compara la cifra con otros países de América, los valores son altamente superiores al promedio (Alonso, 2005). Esto conlleva a un incremento en los estándares para contratar profesionales, a quienes se les exige competencias altamente especializadas (Castro Solano, 2004) lo que dificulta aún más la inserción del recién graduado.

Sumado a esto, las carreras humanísticas han tenido un importante crecimiento en el último tiempo, la mayoría de los estudiantes universitarios optan por carreras del área de las ciencias sociales o las humanidades y presentan mucho menos interés por las ciencias básicas y las carreras técnicas (Jazami \& Sánchez Martínez, 2001). Centrándose específicamente en la carrera de psicología, se observa un incremento de la matrícula de un $40 \%$ en las unidades académicas del país (Pancenza, 1997). Y un crecimiento en la cantidad de universidades que ofrecen la carrera de Psicología en la Argentina, son cuarenta universidades, treinta privadas y diez públicas que cuentan con títulos habilitantes (Alonso \& Gago, 2008). Además el campo de mayor inserción profesional es el área clínica, como profesional independiente, donde existe una saturación de psicólogos y en el último tiempo se produjo una disminución de consultas particulares incrementándose las institucionales o mediante sistemas de reintegro (Borrás \& Bucci, 2003; Pancenza, 1997).

Lo detallado anteriormente incrementa la necesidad de conocer la situación de los graduados, profundizando las investigaciones en este campo para poder formar al estudiante conociendo las demandas y las competencias requeridas por el mercado laboral. 


\section{El Perfil ocupacional del Psicólogo Argentino}

El perfil tradicional del psicólogo en nuestro país ha estado orientado predominantemente hacia el campo de la clínica y la temática general de la salud mental, con una tradición hegemónica del paradigma psicoanalítico (Vezzetti, 1996). Las especializaciones iniciales han sido: clínica, educacional, laboral y forense (Alonso, 1997).

Investigaciones que relevaron la situación de los primeros graduados en psicología en Argentina, observaron en relación a su inserción laboral que casi la totalidad de la muestra se desempeñaba en el área clínica y con menor frecuencia en docencia, psicología educacional, laboral, orientación vocacional, psicohigiene y psicología institucional. Un alto porcentaje de los encuestados trabajaba en más de un área. De lo que también se desprende que la gran mayoría de los psicólogos ejercían sus labores en el área clínica, junto con otro campo profesional (Litvinoff \& Gomel, 1975).

En los últimos años se ha producido una progresiva inclusión de nuevos paradigmas, que han enriquecido el rol profesional. Posiblemente, esta apertura estuvo motivada por las demandas del medio social, las oportunidades laborales y las posibilidades de respuesta de las universidades. Actualmente, los alumnos tienen más opciones teóricas y técnicas. En nuestro país reciben cada vez más atención áreas de la Psicología Social, Comunitaria, de la Salud, de las Organizaciones, Política, con una paulatina apertura a otros modelos teóricos: sistémicos, gestálticos, cognitivos, humanísticos e integrativos (Alonso, 1997).

Como mencionan varios estudios, el trabajo de los jóvenes graduados se caracteriza por ser de pocas horas, en lugares diferentes y con distintas funciones. Se desenvuelven en el ámbito público tanto como en el privado, muchos psicólogos trabajan como profesionales independientes. Manteniéndose el predominio del sexo femenino (Antman, 2002; Borinsky, 2002; Borrás \& Bucci, 2003; Litvinoff \& Gomel, 1975; Pancenza, 1997).

Antman (2003) realizó un estudio sobre las representaciones del quehacer profesional del psicólogo y su inserción laboral en el ámbito de la Salud Mental. Halló una inscripción anual de 1.083 psicólogos para hacer el examen de residencia, un altísimo número considerando que anualmente se reciben alrededor de 2.073 profesionales. Estos datos marcan la importancia del área clínica como el quehacer profesional dominante.

Teniendo en cuenta que aún en nuestros días continúa teniendo predominancia el ámbito clínico, se plantea que aún es hegemónico el perfil del Psicólogo Clínico, de práctica similar a sus comienzos. Donde existen mínimos o nulos espacios de debate acerca de inserción profesional del psicólogo (López, 1999).

Un estudio reciente realizado por Alonso y Gago (2008) muestra la disparidad en la inserción de los psicólogos, señalando que un 60 a 90\% de los profesionales se dedican a la especialidad de la clínica, de un 14 a un $25 \%$ al sector educacional, entre un 1 y un $11 \%$ al campo forense y por último 1 a 5\% en el ámbito de la Psicología laboral. La variación en los porcentajes se debe a diferencias según provincia.

Tal como menciona Saforcada (2008) un amplio campo de la profesión ha sido descuidado, dejando lugar a otros profesionales, inclusive permitiendo el surgimiento de una gran cantidad de instituciones terciarias; cuyo único requisito de ingreso a carreras como Psicología Social o Counselor, es tener finalizados los estudios primarios. En algunos casos, sus egresados compiten por el mismo campo laboral que el psicólogo. Esta fuerte predominancia del ámbito clínico como área de inserción profesional, no es común a todos los países, prueba de ello son las más de 50 divisiones de áreas que son reconocidas por la American Psychological Association.

Una de las características propias del trabajo del psicólogo es su desempeño profesional en más de un área, la mayoría trabaja en el área clínica, además de alguna otra. Situación que no ocurre en otras profesiones, donde se da el hecho contrario que es la especialización. A lo que se le adjudica dos causales diferentes, por un lado, es el rol social poco claro, puesto que el medio espera de ellos que estén capacitados tanto para atender un trastorno mental como para realizar un proceso de orien- 
tación vocacional, por otro lado la necesidad económica, dando la necesidad de tener varios trabajos para sustentarse económicamente (Litvinoff \& Gomel, 1975).

Pancenza, (1997) señala diferentes estrategias de inserción laboral en la práctica del psicólogo. Las cuales se desarrollan a continuación. I) Estrategia única: se refiere al grupo de profesionales que se desempeñan en una única práctica profesional, clínica o laboral, educacional, etc. II) Estrategia diversificada: son los graduados que se encuentran inmersos totalmente en el campo profesional ejerciendo diferentes tareas propias del psicólogo, los que realizan mas de uno de estos quehaceres: pericias, psicología laboral, clínica, docencia universitaria y psicología escolar. III) Estrategia intra y extra campo: este grupo se caracteriza por estar trabajando parcialmente en el campo y desarrolla una tarea que no es competencia especifica del psicólogo: maestro, administrativo u otra profesión, pero paralelamente alguna de estas actividades propias del perfil del psicólogo. Y por último IV) Estrategia innovadora: integran este grupo aquellos egresados que crearon un espacio donde previamente no existía la demanda de psicólogos.

\section{Método}

\section{Objetivo General}

- Analizar la inserción laboral de una muestra de psicólogos egresados durante el período 2003-2008, de la Licenciatura en Psicología de una Universidad de gestión privada.

\section{Objetivos Específicos}

- Delimitar cuáles son las áreas de inserción laboral del joven profesional.

- $\quad$ Establecer el tipo de inserción laboral del graduado de psicología.

- Conocer acerca de la continuación con estudios de postgrado

\section{Muestra}

Estuvo conformada por 70 graduados de psicología de una Universidad de gestión privada. El 82.85\% de los encuestados era de sexo femenino, la edad de los egresados oscilaba entre los 22 y los 65 años de edad, con una media de 35 años. Los profesionales se habían graduado entre los años 2003 y 2008.

\section{Instrumento}

Con el objetivo de realizar el seguimiento de la trayectoria laboral de los jóvenes graduados en Psicología se diseñó un instrumento para conocer diversos aspectos de inserción laboral y de la formación de posgrado.

\section{Procedimiento}

Se administró una encuesta de manera anónima, voluntaria e individual. La encuesta se envío vía email a los egresados de la carrera de Psicología. Se consideró que este era un buen sistema ya que muchos graduados se encuentran viviendo en otras ciudades e inclusive en otros países.

Para ello se recurrió a una base de datos actualizada conteniendo las direcciones de e-mail de los graduados de las distintas cohortes. Desde un comienzo se supo que no se podría llegar a todos los graduados de la institución porque I) no se contaba con los correos electrónicos de algunos de ellos (especialmente de los que terminaron sus estudios hace muchos años) o no se tenían las direcciones actualizadas de todos; II) los relevamientos vía correo arrojan una baja tasa de respuesta.

En estas condiciones se envió un cuestionario (ver Instrumento) y se otorgó un plazo de cuatro meses para recibir las devoluciones.

El instrumento cuenta con algunos ítems que requieren un análisis cualitativo, como es el caso del área de inserción y actividades realizadas. En estos casos se trabajó con sistema de jueces expertos, tres profesores de la Universidad que trabajan en la temática leyeron y calificaron las respuestas. 


\section{Resultados}

Los resultados serán presentados en tres apartados, I) primeramente se describirá la distribución de la muestra en cuanto al tiempo requerido para insertarse como psicólogo y el modo de hacerlo, II) se desarrollarán los ámbitos de inserción profesional, III) se detallará la continuación con la formación de posgrado.

\section{I) La inserción profesional}

Los resultados hallados en relación a la inserción laboral de los graduados permiten determinar que el 82.85 \% de los egresados trabaja como psicólogo y el 17.14 \% aún no, del porcentaje que no se desempeña en este campo, el 75 \% hace menos de un año y medio que se ha graduado. El tiempo promedio en obtener su primer empleo fue de 3.86 meses.

El 39\% de los jóvenes egresados se contactó con la profesión antes de haber concluido sus estudios, realizando prácticas pre-profesionales supervisadas. De los cuales el 25 \% se encuentra desempeñando su función en la institución donde realizaron su práctica pre-profesional.

De los que se desempeñaron dentro de la psicología antes de graduarse: ya sea mediante la realización de prácticas pre-profesionales o insertándose a trabajar en el período que va desde la finalización del cursado hasta la obtención del título de grado, el 47.8\% lo hizo en el área Clínica, $30.4 \%$ en Laboral y el 21.7\% en Educacional.

\section{Gráfico 1. Áreas de inserción pre-profesional}

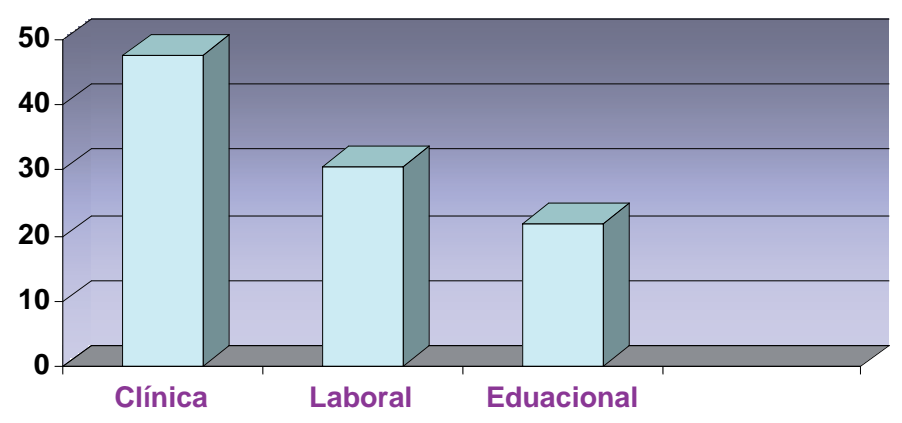

\section{II) Ámbito de inserción}

En cuanto al área de inserción, hay que mencionar que el 12\% de los encuestados se desarrolla profesionalmente en más de un área, siendo generalmente el área principal de desarrollo la clínica y sumando a este otro ámbito de desempeño profesional. Se trata de elecciones no excluyentes y es por ello que los resultados que se presentan a continuación, están expresados en porcentajes y exceden el valor de cien. El 72.41\% de los graduados se desempeña profesionalmente en el área clínica. De los cuales 76\% (32) poseen consultorios particulares, 30.95\% (13) trabajan en Centros Privados y 17.24\% (10) en Hospitales Públicos. De lo que también se desprende que el 54\% de la muestra se desempeña en más de un ámbito dentro del área clínica, alternado consultorio particular, con centros privados u hospitales.

En relación a las otras áreas profesionales: el 14\% (8) se inserto en el ámbito Educacional, el 27.5\% (16) en el área Laboral, el 1.72\% (1) en el área Forense, 1.72 \% (1) en el ámbito de la psicología Social y Comunitaria, el 1.72 \% (1) en el área exclusivamente Académica. 


\section{Gráfico 2. Ámbitos de Inserción profesional}

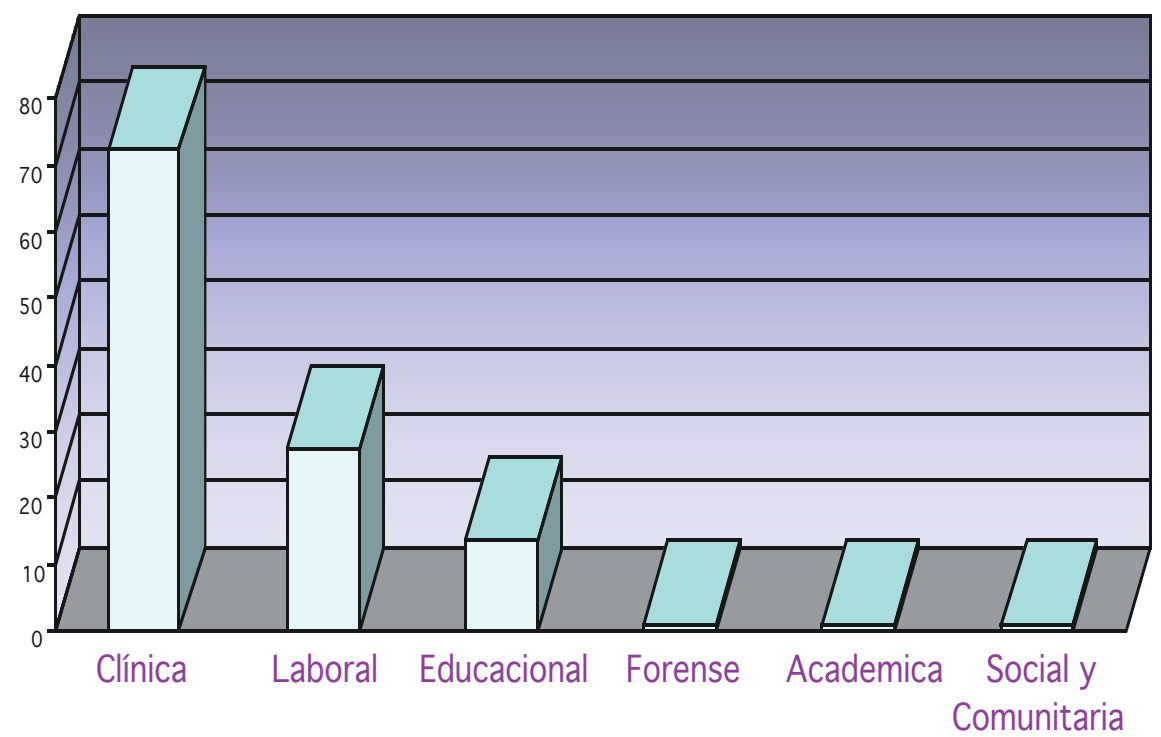

Se les solicitó a los graduados que comentaran cuales eran las actividades que realizaban con mayor frecuencia dentro de su ámbito laboral. Cabe destacar que por el reducido número de la muestra, la descripción es acotada y no se extraerá ninguna conclusión de las mismas, ya que en algunas áreas solo se contó con la respuesta de un graduado.

Tabla 1. Actividades principales según área de inserción

\begin{tabular}{ll}
\hline $\begin{array}{l}\text { Área Profesional } \\
\text { Perfil }\end{array}$ & Actividades principales \\
\hline Clínica & $\begin{array}{l}\text { Tratamiento a niños, adolescentes y adultos } \\
\text { Psicodiagnóstico } \\
\text { Evaluación y rehabilitación Neuropsicológica } \\
\text { Entrevistas y psicotécnicos } \\
\text { Evaluación de potencial } \\
\text { Selección de personal } \\
\text { Social y comunitaria }\end{array}$ \\
Educacional & $\begin{array}{l}\text { Tareas de gabinete } \\
\text { Entrevistas a padres y alumnos }\end{array}$ \\
Académico & $\begin{array}{l}\text { Investigación } \\
\text { Dictado de clases }\end{array}$ \\
Forense & Asistencia a la persona privada del libertad \\
& Investigación \\
\hline
\end{tabular}




\section{III) Formación de Posgrado}

En cuanto a la formación de posgrado se puede observar que una importante proporción, el 60\% de la muestra evaluada continúa con su formación una vez finalizados los estudios de grado.

En cuanto al tipo de formación elegida se aprecia, al menos dentro de los primeros años de graduación, una mayor inclinación hacía una formación reducida en el tiempo. La mayoría realizan Carreras de especialización o Cursos de posgrado (90.47 \%), son muy pocos los casos en los que han continuado con una formación en Docencia Universitaria (7.14 \%), en una proporción mucho menor han optado por la realización de un Doctorado (2.38 \%) y ninguno ha iniciado una formación de Maestría.

En cuanto a la elección del lugar para realizar la formación de Posgrado, ocupa el primer lugar en la elección de los graduados los Centros privados (43.9\%), en un segundo lugar las instituciones Universitarias (41.46 \%) y por último la formación en Hospitales públicos (12.19\%).

\section{Gráfico 3. Lugar elegido para la formación de Posgrado}

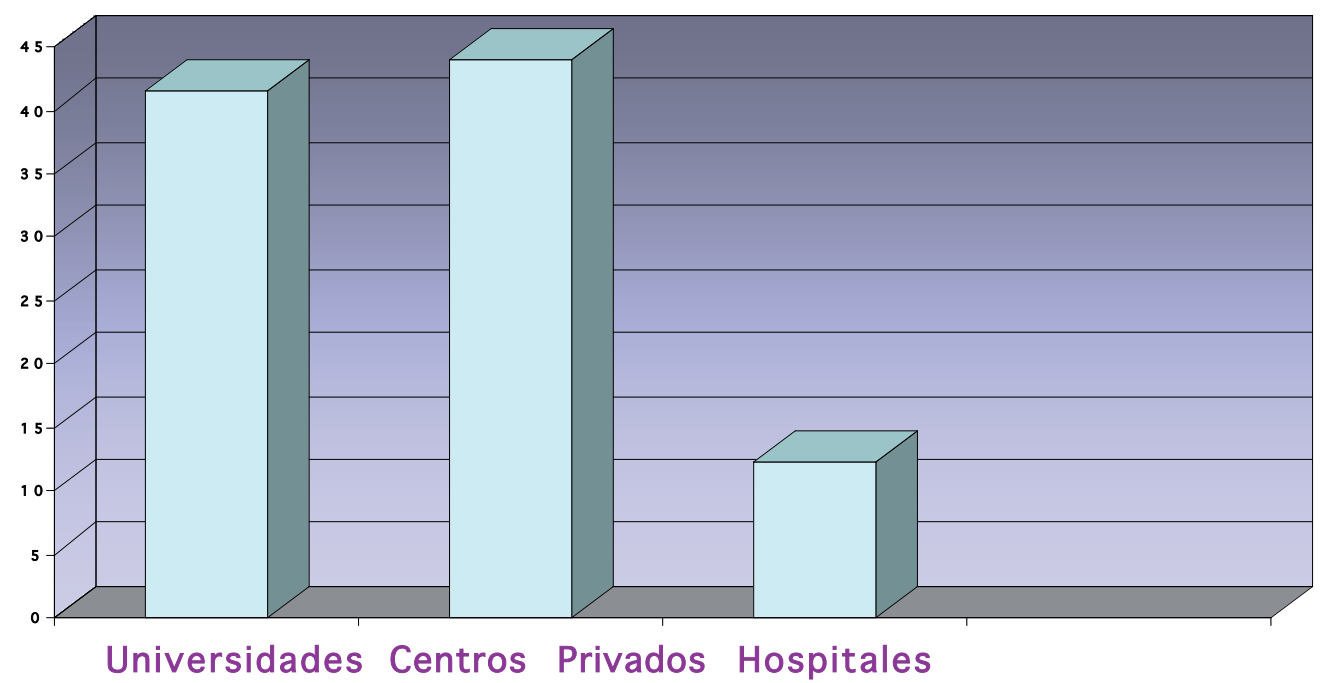

Por último en cuanto al área de formación, realizan predominantemente posgrados centrados en modelos psicoterapéuticos, con predominancia del modelo cognitivo-conductual, luego la terapia sistémica y por último el psicoanálisis. La elección de temas para cursos de mayor especificidad es sumamente variada: desde Psicología perinatal, Prevención de suicidio, MDR, Arte y Psicología, Neuropsicología, entre otras.

\section{Discusión}

La presente investigación consiste en un primer análisis de la relación entre educación, formación y empleo de los egresados en psicología de una universidad de gestión privada. Tal como se ha dicho, la inserción laboral varía acorde al momento histórico y el contexto. Se sabe que en los últimos años el mercado laboral ha cambiado y las crisis recientes han hecho más difícil aún la inserción de los graduados. Actualmente la educación universitaria ya no garantiza en ingreso al mercado laboral. Es por ello que resulta fundamental realizar este tipo de estudios, para conocer cual es la realidad de los graduados recientes, cuales son las áreas de mayor interés y cuales las de mayor demanda, cuales son las dificultades con las que se encuentra a la hora de buscar trabajo, cuales sienten que son sus fortalezas y su debilidades. 
Debido a que la inserción del profesional recién graduado se hace dificultosa, una de las preguntas que sigue vigente es: si el nivel de desempleo podría modificarse si tal como propone Pancenza (1997) la Universidad adaptara los contenido curriculares a los cambios en el mercado productivo. También debe considerarse qué sucedería si en la Argentina, como pasa en muchos países, la orientación profesional se definiera por cupos y éstos estuvieran determinados por la demanda social del Psicólogo en cada área. Estas preguntas no pueden responderse a partir del presente estudio, pero el mismo intenta aportar datos para poder implementar en un futuro cercano diferentes estrategias para reducir el nivel de desempleo.

Los resultados obtenidos en este estudio permiten arribar a una serie de conclusiones.

En Argentina al igual que en Brasil, Chile, Colombia, México y casi toda Latinoamérica, la inserción laboral está fuertemente orientada al área clínica. Continúa el área laboral y educacional, por último el ámbito forense. Esta distribución se ha mantenido tradicionalmente de este modo. A pesar de ello en otros lugares, principalmente Europa y Estados Unidos, la distribución es más pareja, llegando en algunos caso a tener mayor cantidad de Psicólogos en el área institucional/organizacional que en el área clínica.

Como es frecuente dentro de la profesión del Psicólogo, la mayoría de los encuestados realiza más de un trabajo, dándose el pluriempleo, cuestión que se da desde los primeros graduados en nuestro país. Históricamente en la Argentina el Psicólogo ha trabajado predominantemente en forma independiente y bajo condiciones de Pluriempleo. Es frecuente que aunque se desempeñe en una sola área, trabaje en más de una institución. Asistiendo pocas horas a varios lugares. Este es un fenómeno poco común en otras profesiones donde el trabajo es necesariamente de tiempo completo. Estos resultados han sido coincidentes con estudios anteriores presentados al inicio de este trabajo (Antman, 2002; Borinsky, 2002; Borrás \& Bucci, 2003; Litvinoff \& Gomel, 1975; Pancenza, 1997).

Se han hallado algunas diferencias con el estudio presentado por Alonso y Gago (2008) la principal es en la inserción en el área Laboral, el estudio señalado halló una inserción del 1 a 5\% y en este trabajo se encontraron valores muy superiores, 27,5\%, incluso más elevados que la inserción en el área Educacional y bastante más que el ámbito Forense. De todos modos se debe recordar que por el tamaño de la muestra y el hecho de haber sido tomada en una sola Universidad, tiene altas probabilidades de tener algún sesgo.

En cuanto a la estrategia de inserción utilizada, según la clasificación de Pancenza (1997) se puede decir que en esta muestra es predominantemente diversificada: inserción en más de un campo profesional. De todos modos futuros estudios deberían realizar un análisis más exhaustivo de las diferentes modalidades de inserción.

En cuanto a la formación de Posgrado, es destacable la importancia que le otorgan los egresados a continuar su formación académica, siendo más de la mitad de la muestra quienes realizan algún tipo de especializaciones dentro de los primeros 5 años de graduación. Este fenómeno también puede estar vinculado a la reducción de la formación de grado y la cada vez más necesaria formación de posgrado y formación a lo largo de la vida.

Tal como fue comentado anteriormente, muchos graduados han continuado su formación y/o su trabajo en el lugar donde realizaron su práctica Pre-profesional. Por este y por otros aspectos, se considera importante la inclusión de prácticas pre-profesionales durante la formación de grado, ya que las mismas otorgan la posibilidad de una aproximación gradual al quehacer profesional, acotando las distancias entre la formación teórica y la práctica profesional.

Esta formación permite que el estudiante esté en contacto con profesionales observando su práctica desde la formación de grado, que adopte modelos a seguir. Posibilita que el estudiante a lo largo de su formación ponga en juego diferentes roles, se imagine en diferentes ámbitos del quehacer profesional y vea a los profesionales en el ejercicio de su práctica. Esta aproximación gradual tiene 
varios beneficios, I) minimiza las posibilidades de elecciones de inserción profesional erróneas, ya que durante la formación de grado el estudiante se va imaginando y va conociendo diferentes campos de inserción.

II) amplia las posibilidades de elección de campos de actuación ya que en algunos casos la elección del ámbito clínico está fuertemente basada en una formación de grado orientada a la clínica y el desconocimiento de otros campos posibles de acción.

III) facilita la inserción ya que el joven graduado realizó aproximaciones graduales, supervisadas y por lo tanto el impacto de la inserción profesional es menor.

Es dable destacar que este estudio es acotado, que la muestra es pequeña y por ello ha sido difícil analizar algunas variables. Sería deseable continuar profundizando esta línea de investigación, ampliando la muestra, haciéndola heterogénea en cuanto a Universidad de graduación para evitar el sesgo. Asimismo sería recomendable la inclusión de otras variables como ser: grado de satisfacción con el trabajo, mayor detalle de las tareas realizadas, y otras que no han sido evaluadas en este estudio.

A modo de cierre, se puede concluir que en el caso de la profesión del psicólogo, es fundamental que la formación otorgue las herramientas necesarias para poder lograr una mejor inserción y que la misma desarrolle y forme a los estudiantes en la diversidad de campos de acción profesional.

\section{Referencias}

Alonso, M. M. (2005). Los psicólogos en la Argentina. Psicodebate 6. Psicología, Cultura y Sociedad. 7-14.

Alonso, M. M. \& Gago, P. T. (2008). Algunos aspectos cuantitativos de la evolución de la Psicología en la Argentina 1975-2005. Universidad de Buenos Aires Facultad de Psicología. Disponible en http://www.modestoalonso.com.ar/

Alonso, M. M; \& Nicenboim, E. (1997). La psicología en la republica Argentina: Aspectos académicos y profesionales, Papeles del psicólogo, 67, 71-75.

Antman, J. (2002). El trabajo ad-honorem en los psicólogos. Un acercamiento a la inserción profesional. Proyecto: Representaciones Sociales acerca del quehacer Profesional del Psicólogo: el caso de las Concurrencias de Salud Mental de la Ciudad Autónoma de Buenos Aires. Departamento IV, Ciclo Básico Común, Universidad de Buenos Aires.

Antman, J. (2003). La inserción del psicólogo recién graduado: el examen de residencia y las representaciones del quehacer profesional. Proyecto: Representaciones Sociales acerca del quehacer Profesional del Psicólogo: el caso de las Concurrencias de Salud Mental de la Ciudad Autónoma de Buenos Aires. Departamento IV, Ciclo Básico Común, Universidad de Buenos Aires.

Ardila, R. (2004). La Psicología Latinoamericana: El primer Medio Siglo. Revista Interamericana de psicología (38), 2, 317-322..

Banco Mundial (1994). Higher Education: The Lessons of Experience. Washington, D.C.: The World Bank.

Borinsky, M. (2002). Las primeras estrategias de inserción profesional de los psicólogos, IX Jornadas de Investigación de la Facultad de Psicología de Universidad de Buenos Aires.

Borrás, G. \& Bucci, I. (2003). Educación, trabajo y perspectiva de género: profesionales y estudiantes avanzados de la carrera de psicología. IV Congreso Nacional de estudios del Trabajo. Facultad de Ciencias Económicas de la Universidad de Buenos Aires. 
Casali, C; Farber, A; Giangiácomo, G; Hernández, G. \& Toribio, D. (2005). Cuestiones de Educación Superior. Buenos Aires: Universidad Nacional de Lanús.

Castro Solano, A. (2004). Las competencias profesionales del psicólogo y las necesidades de perfiles profesionales en los diferentes ámbitos laborales, Interdisciplinaria, 21,(2):117-152.

Gómez, M. (2000). El mercado de trabajo para los egresados universitarios recientes. Resultados de una encuesta de inserción ocupacional de algunas especialidades profesionales. Buenos Aires: Editorial de la Universidad Nacional de Tres de Febrero.

Huerta González, T; Valenzuela, B. \& Rodríguez Llanes, P. (2007). Identificación de exigencias profesionales de los egresados de la licenciatura en psicología de la Universidad de Sonora desde el enfoque de los empleadores. Revista Email Educativo, Universidad Nacional de Colombia (1). Versión electrónica. Disponible en: http://www.revistas.unal.edu.co/index.php/email/article/ view/1309

Isorini, .M. E. Cerro .M. E. \& Goni. J. (2006). Un estudio comparativo del comportamiento laboral de los graduados en Ciencias Sociales y de las Ingenierías de la Universidad Nacional de Santiago del Estero. $7^{\circ}$ Congreso Nacional de Estudios del trabajo.

Jazami, A. Y \& Sánchez Martínez, E (2001). Estudiantes y profesionales en la Argentina. Una mirada desde la encuesta permanente de hogares. Buenos Aires: Editorial de la Universidad Nacional de Tres de Febrero.

Linn, H. (2007). La situación laboral del psicólogo en Chile. VII Congreso Nacional de Psicología, Santiago de Chile.

Litvinoff, N. \& Gomel, S. (1975). El psicólogo y su Profesión. Buenos Aires: Nueva Visión.

López, M. (1999). Formación profesional y sociedad de fin de siglo. En A. M. Fernández. Instituciones Estalladas. Buenos Aires: EUDEBA.

Miranda Zapata, E. \& Sutulov Baez, C. (2007). Caracterización del perfil del psicólogo de la ciudad de Valdivia. VII Congreso Nacional de Psicología, Santiago de Chile.

Moura, E. P. G. (2003) A psicologia (e os psicólogos) que temos e a psicologia que queremos. Disponible en http://www.pol.org.br/pesquisa/resposta_pesquisa.cfm?id_area

OECD (1992). From Higher Education to Employment. Paris: OECD.

OECD (1994). From Higher Education to Employment: Synthesis Report. Paris: OECD.

Ortega Álvarez, M. L. (1982). Modelos de Comportamiento para el joven psicólogo. Papeles del psicólogo, 2. Versión electrónica http://www.papelesdelpsicologo.es/vernumero.asp?id=31

Pancenza, M. I. (1997). Tipología de la inserción laboral de los psicólogos: Estrategias y prácticas laborales. Universidad Nacional de Mar del Plata. Proyecto Universidad y mercado de trabajo.

Pedroza Flores, R; Villalobos Monroy, G. \& Morales Euzarraga, M. (2007). Inserción laboral y trayectorias vitales del egresado en Psicología. México: Universidad Autónoma del Estado de México.

Peiró, J. M. (1991). Psicología de la Organización. Madrid: UNED.

Richino, S. V. (1985). Estructuración del rol profesional: laboratorio en procesos institucionales. Buenos Aires: Ediciones Kergieman.

Riquelme, G. C. \& Herger, N. (2002). La transición de la educación al trabajo de los estudiantes avanzados de tres universidades argentinas. III Congreso Nacional y I Encuentro Latinoamericano de Estudios Comparados en Educación.

Rojas, V. (2007). Inserción laboral de psicólogos titulados por la universidad La República. VII Congreso Nacional de Psicología, Santiago de Chile. 
Saforcada, E. (2008). La Psicología en la Argentina: desarrollo disciplinar y realidad nacional. Interamerican Journal of Pschology 42 (3).

Testa, J \& Spinoza, M. (2005). Hacia la construcción de la red de trabajo sobre saberes, educación y formación para el trabajo. III Congreso Nacional y I Encuentro Latinoamericano de Estudios Comparados en Educación.

Verdugo-Lucero J. C; Monroy-Galindo E. C; Marquez-González, C.V.\& Ceja-Castillo, M. (2002). La formación profesional de psicólogos: el currículo integrado y aprendizaje centrado en el estudiante (CIACE-PSICOLOGIA), una experiencia diferente. Facultad de psicología. Universidad de Colima. Disponible en: www.lie.upn.mx/docs/docinteres/CIACE.pdf

Vezzetti, H.(1996). Los estudios históricos de la psicología en la Argentina. Cuadernos Argentinos de Historia de la Psicología, San Luis, (2).

Weisgras, S. (2006). Determinantes de la sobre educación de los jóvenes en el mercado laboral Argentino. VII Congreso Nacional de estudios de trabajo. 


\section{ANEXO}

\section{Encuesta para graduados}

En esta encuesta Usted encontrará una serie de preguntas o frases para completar. Le pedimos que responda de la forma más sincera posible. Recuerde que no existen respuestas correctas o incorrectas. Intente no dejar preguntas sin responder. Le recordamos que la participación en este estudio es voluntaria y que los datos serán tratados con total confidencialidad.

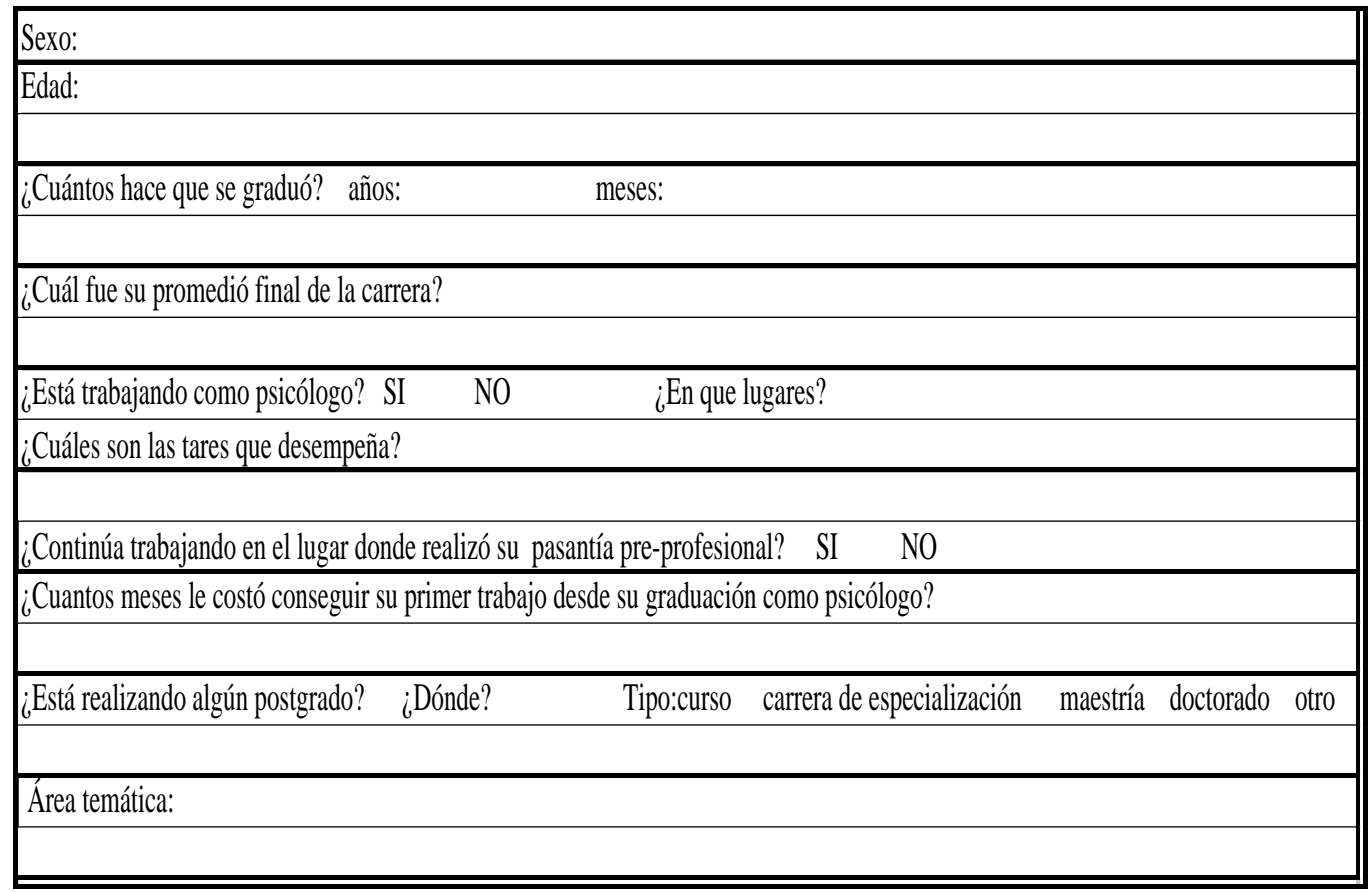

